Pathologe 2021 · 42:197-207

https://doi.org/10.1007/s00292-021-00925-w

Angenommen: 28. Januar 2021

Online publiziert: 24. Februar 2021

(c) Springer Medizin Verlag GmbH, ein Teil von

Springer Nature 2021

Schwerpunktherausgeber

W. Roth, Mainz

P. Boor, Aachen

\section{Zusatzmaterial online}

Zusätzliche Informationen sind in der Online-Version dieses Artikels (https:// doi.org/10.1007/s00292-021-00925-w) enthalten.

Die COVID-19-Pandemie stellt die globalisierte Gesellschaft vor eine bislang ungekannte Herausforderung. Gesellschaftliche und medizinische Fragen können nur durch möglichst exakte Kenntnisse der Biologie des Virus und der Auswirkungen auf den befallenen Organismus beantwortet werden. Der Nutzen autoptisch gewonnener Erkenntnisse, der zu Beginn der Pandemie nicht richtig eingeschätzt wurde, steht jetzt allerdings außer Frage. Im deutschsprachigen Raum wurden an mehreren Zentren sehr früh systematische autoptische Untersuchungen von an COVID-19 Verstorbenen durchgeführt, deren

Die Reihung der Autoren erfolgt in alphabetischer Reihenfolge.

Die Autoren P. Eichhorn, S. F. Lax, T. Menter, J. Slotta-Huspenina, S. von Stillfried und A. Tzankov haben federführend und in gleicher Weise zu diesem Artikel beigetragen.

Peter Boor ${ }^{1}$ Philip Eichhorn ${ }^{2}$ Arndt Hartmann ${ }^{2}$ Sigurd F. Lax ${ }^{3,4} \cdot$ Bruno Märk $^{5,8}$. Thomas Menter ${ }^{6} \cdot$ Kristijan Skok $^{3}$. Julia Slotta-Huspenina ${ }^{7} \cdot$ Saskia von Stillfried ${ }^{1}$. Alexandar Tzankov ${ }^{6} \cdot$ Gregor Weirich $^{7}$

${ }^{1}$ Institut für Pathologie, Universitätsklinikum Aachen, RTWH Aachen, Aachen, Deutschland

${ }^{2}$ Institut für Pathologie, Universitätsklinikum Erlangen, Erlangen, Deutschland

${ }^{3}$ Institut für Pathologie, Landeskrankenhaus Graz II, Akademisches Lehrkrankenhaus der Medizinischen Universität Graz, Graz, Österreich

${ }^{4}$ Medizinische Fakultät, Johannes-Kepler-Universität Linz, Linz, Österreich

${ }^{5}$ Allgemeine und Spezielle Pathologie, Medizinische Fakultät, Universität Augsburg, Augsburg, Deutschland

${ }^{6}$ Institut für Medizinische Genetik und Pathologie, Universitätsspital Basel, Universität Basel, Basel, Schweiz

${ }^{7}$ Institut für Pathologie und pathologische Anatomie, Technische Universität München, München, Deutschland

${ }^{8}$ Institut für Pathologie und Molekulare Diagnostik, Universitätsklinikum Augsburg, Augsburg, Deutschland

\title{
Praktische Aspekte von COVID-19-Obduktionen
}

\section{Ergebnisse von außerordentlich hohem wissenschaftlichem Wert sind.}

\section{Obduktionen als Möglichkeit, neue Erkrankungen besser zu verstehen}

Obduktionen bieten die einzigartige Möglichkeit, die unterschiedlichen Verlaufsformen einer Erkrankung, aber auch wenig bekannte und neuartige Krankheiten genau zu untersuchen. Dabei können Organveränderungen studiert und Rückschlüsse auf Pathomechanismen gezogen werden. Im Falle von COVID-19 waren es vor allem Institute aus dem deutschsprachigen Raum, die in Europa die ersten systematischen Obduktionen von an COVID-19-Erkrankten durchführten [1-3] und deren Ergebnisse rasch publizierten. $\mathrm{Zu}$ diesem Zeitpunkt gab es keine publizierten Ergebnisse systematisch aufgearbeiteter Obduktionsserien, nur einzelne Fälle und Operationspräparate von Lungen sowie bioptische Studien an Verstorbenen [4-6]. Auf Basis der Obduktionen entstanden außerdem innovative grundlagenorientierte wissenschaftliche Arbeiten [7-9], die wesentliche
Erkenntnisse zur Pathophysiologie von COVID-19 erbracht haben, beispielsweise, dass ein diffuser alveolärer Schaden (DAD) beider Lungen im Vordergrund der Organschädigung steht, der offenbar unabhängig von der Durchführung einer invasiven Beatmung auftritt, und häufig mit thrombotischen Verschlüssen von Pulmonalarterienästen auf segmentaler und subsegmentaler Ebene $[1,2,10]$ und einer Endotheliitis einhergeht [8]. Die Schädigung anderer Organe ist möglicherweise als Folge eines protrahierten Schockgeschehens mit nachfolgendem Multiorganversagen zu betrachten und kann mitunter auch nur in geringem Ausmaß vorhanden sein. Dazu zählen die ischämische Tubulusschädigung der Nieren [1, 11-13], fokale Pankreatitiden [1] und eine fokale ischämische Kolopathie [14]. Ergebnisse von Analysen des zentralen Nervensystems (ZNS) sind widersprüchlich und liefern bislang keine ausreichenden Beweise für eine direkte Schädigung des Gehirns durch SARSCoV-2 mit Ausnahme einer Einbeziehung in systemische Gerinnungsprozesse [15-19].

In Deutschland wurde früh anlässlich der ersten Infektionswelle das Deutsche Register für COVID-19-Obduktionen 


\section{Schwerpunkt: COVID-19}

Tab. 1 Darstellung dervom Robert Koch-Institut (RKI) empfohlenen und in den Zentren durchgeführten Schutzmaßnahmen - technische Kennzahlen, persönliche Schutzausstattung und obduktionstechnische Aspekte

\begin{tabular}{|c|c|c|c|c|c|c|c|}
\hline & $\begin{array}{l}\text { Empfeh- } \\
\text { lung RKI }\end{array}$ & Empfehlung Aachen & Augsburg & Erlangen & $\begin{array}{l}\text { TU Mün- } \\
\text { chen }\end{array}$ & Graz & Basel \\
\hline $\begin{array}{l}\text { Anzahl der Mitar- } \\
\text { beiter pro Obdukti- } \\
\text { on (Ärzte [Ä]/Sekt.- } \\
\text { Assistenten [PA]) }\end{array}$ & - & $1 A / 2 P A$ & $4 \ddot{A}$ & $2 \ddot{A} / 1 P A$ & $2 \ddot{A} / 1 P A$ & $2 \ddot{A} / 1 P A$ & $2 \ddot{A} / 0-1 P A$ \\
\hline \multicolumn{8}{|c|}{ Persönliche Schutzausrüstung und -Sektionsraumausstattung } \\
\hline $\begin{array}{l}\text { Sektionssaal mit } \\
\text { Unterdruckverhält- } \\
\text { nissen }\end{array}$ & - & $\mathrm{X}$ & $X$ & $\mathrm{X}$ & $\mathrm{X}$ & $X$ & $\mathrm{x}$ \\
\hline $\begin{array}{l}\text { Luftwechselraten } \\
\text { (pro Stunde) }\end{array}$ & - & $\geq 12 \mathrm{fach}$ & $13 \mathrm{fach}$ & $\geq 12$ fach & $\geq 12$ fach & $20 \mathrm{fach}$ & 9fach \\
\hline $\begin{array}{l}\text { N95-Maske/FFP2/3- } \\
\text { Maske }\end{array}$ & $\mathrm{x}$ & $\mathrm{x}$ & $x$ & $\mathrm{X}$ & $x$ & $x$ & $\mathrm{x}$ \\
\hline Augenschutz & $x$ & $x$ & $x$ & $x$ & $x$ & $x$ & $x$ \\
\hline Kopfhaube & - & $x$ & $x$ & $x$ & $x$ & $x$ & $x$ \\
\hline Mantel & $x$ & $x$ & $x$ & $x$ & $x$ & $x$ & Gefahrgutanzug \\
\hline Handschuhe & $\begin{array}{l}X \text { (mindes- } \\
\text { tens ein } \\
\text { Paar) }\end{array}$ & X (doppelt) & X (doppelt) & $\begin{array}{l}X \text { (dop- } \\
\text { pelt) }\end{array}$ & $\begin{array}{l}X \text { (dop- } \\
\text { pelt) }\end{array}$ & X (doppelt) & X (doppelt) \\
\hline $\begin{array}{l}\text { Schnittfeste Hand- } \\
\text { schuhe aus synthe- } \\
\text { tischem Netzgewe- } \\
\text { be/Kevlar }\end{array}$ & - & $\mathrm{X}$ & $x$ & - & - & $x$ & - \\
\hline $\begin{array}{l}\text { Wasserdichte } \\
\text { Schürze }\end{array}$ & $x$ & $\mathrm{X}$ & $x$ & $x$ & $X$ & $x$ & $x$ \\
\hline $\begin{array}{l}\text { Gummischuhe/- } \\
\text { stiefel }\end{array}$ & $x$ & $x$ & $x$ & $X$ & $x$ & $x$ & $x$ \\
\hline $\begin{array}{l}\text { Unter PPE: OP- } \\
\text { Hemden, Hosen }\end{array}$ & - & $x$ & $x$ & $x$ & $x$ & $x$ & $x$ \\
\hline $\begin{array}{l}\text { Obduktion im Bo- } \\
\text { dybag }\end{array}$ & - & $x$ & $x$ & $x$ & $x$ & b & - \\
\hline $\begin{array}{l}\text { Gewebefixations- } \\
\text { zeiten in Formalin } \\
\text { (in Stunden) }\end{array}$ & - & $336-504$ & 96 & 48 & 72 & $24^{c} / 72^{c}$ & 72 \\
\hline Sonstiges & - & $\begin{array}{l}\text { 2-stufiges Verfahren, } \\
\text { Organpräparation nach } \\
\text { Formalinfixation (siehe } \\
\text { Zusatzmaterial online, } \\
\text { „Verfahrensanweisung } \\
\text { COVID-19-Obduktionen } \\
\text { der Uniklinik RWTH Aa- } \\
\text { chen“) }\end{array}$ & $\begin{array}{l}\text { Knorpelzange für } \\
\text { Thorax } \\
\text { IQ-Air Healthpro } \\
250 \text { NE Raumluftrei- } \\
\text { niger (IQAir, Gol- } \\
\text { dach, Schweiz) }\end{array}$ & - & $\begin{array}{l}\text { Hand- } \\
\text { bohrer } \\
\text { für Schä- } \\
\text { deltre- } \\
\text { panation }\end{array}$ & $\begin{array}{l}\text { Säge mit Absau- } \\
\text { gung für Schädel, } \\
\text { Knorpelzange für } \\
\text { Thorax } \\
\text { Lungenpräpara- } \\
\text { tion nach For- } \\
\text { malinfixierung }\end{array}$ & $\begin{array}{l}\text { Handsäge für Schädel } \\
2 \mathrm{~h} \text { vor der Autopsie: } 4 \% \\
\text { phosphatgepuffertes } \\
\text { Formalin, instilliert in } \\
\text { Mund, Nase, Pharynx }\end{array}$ \\
\hline \multicolumn{8}{|c|}{$\begin{array}{l}\text { RKI Robert-Koch-Instiut, TUM Technische Universität München, FFP "filtering facepiece", PPE "personal protective equipment" } \\
\text { aLt. RKI sind FFP2-Masken als Mindestausstattung definiert } \\
\text { "Bodybag zum Transport des Leichnams } \\
\text { "Fixierungzeiten in Abhängigkeit der Präparategröße }\end{array}$} \\
\hline
\end{tabular}

(DeRegCOVID, www.DeRegCOVID. ukaachen.de) in enger Zusammenarbeit der Deutschen Gesellschaft für Pathologie (DGP) und des Berufsverbandes der Deutschen Pathologen e. V. (BDP) ins Leben gerufen (offizieller Start am 15.04.2020) [20]. Im Rahmen des Registers wurde eine Verfahrensempfehlung ausgearbeitet, die das Vorgehen am
Universitätsklinikum Aachen abbildet (• Tab. 1) und mit höchster Priorität die Sicherheit der Mitarbeiter gewährleistet (Zusatzmaterial online, „Verfahrensanweisung COVID-19-Obduktionen der Uniklinik RWTH Aachen“). Diese wurde durch BDP und DGP als Beispiel bzw. Hilfestellung allen Mitgliedern zur Verfügung gestellt und ist insbeson- dere für Zentren gedacht, die keine ausgedehnte Erfahrung mit hochinfektiösen Obduktionen haben. Dabei sollten immer alle Prozesse an die lokalen Gegebenheiten angepasst und durch die entsprechenden Stellen geprüft werden, z.B. die hausinterne Krankenhaushygiene, institutsinterne QM-Richtlinien und zentrale Richtlinien für Biologische Si- 
Pathologe $2021 \cdot 42: 197-207$ https://doi.org/10.1007/s00292-021-00925-w

(c) Springer Medizin Verlag GmbH, ein Teil von Springer Nature 2021

P. Boor · P. Eichhorn · A. Hartmann · S. F. Lax · B. Märkl · T. Menter · K. Skok · J. Slotta-Huspenina · S. von Stillfried · A. Tzankov · G. Weirich Praktische Aspekte von COVID-19-Obduktionen

\section{Zusammenfassung}

Hintergrund. Die COVID-19-Pandemie

stellt die medizinische Fachwelt vor eine

bislang ungekannte Herausforderung.

Obduktionen sind für die Erforschung dieser

neuen Krankheit wesentlich, ihre sichere

Durchführbarkeit wurde aber anfangs infrage

gestellt.

Fragestellung. Unter welchen rechtlichen

Rahmenbedingungen und unter welchen

Schutzmaßnahmen können COVID-19-

Obduktionen durchgeführt werden?

Material und Methoden. Das obdukti-

onstechnische Vorgehen in 5 Zentren in

Deutschland, Österreich und der Schweiz

wird unter Berücksichtigung der jeweiligen

rechtlichen Grundlagen und der ergriffenen

Schutzmaßnahmen dargestellt.

Ergebnisse. In allen Institutionen konnten

die Obduktionen in technisch geeigneten
Räumen sicher durchgeführt werden. Die persönliche Schutzausrüstung umfasste Augenschutz, Mund-Nasen-Masken (mindestens FFP2), Kopfhauben, Funktionskleidung, Mäntel, Schürzen und 2 Paar Handschuhe. In 4 Instituten wurden die Leichen nach unterschiedlichen Techniken eröffnet. In einem Institut wurde ein minimalinvasives Verfahren, die postmortale ultraschallunterstützte Gewebeentnahme im Rahmen des "postmortal imaging and biopsy programs", durchgeführt. Letztere gibt zwar keine makroskopischen Einblicke in die inneren Organe, ermöglicht aber eine standardisierte bioptische Gewinnung von Gewebe für Diagnostik und Forschung. Aus den Obduktionen resultierten mehrere Arbeiten in hochrangigen Journalen, die profunde Einblicke in die Organveränderun- gen ermöglichten und wesentliche Schlüsse auf die Pathomechanismen zuließen. VirusRNA konnte häufig in COVID-19-Verstorbenen nachgewiesen werden. Vereinzelt gelang auch die Anzüchtung der Viren. Die Frage nach der postmortalen Infektiosität bleibt aber unklar und bei Ct-Werten über 30 umstritten.

Fazit. Unter Beachtung geeigneter Schutzmaßnahmen sind Obduktionen von COVID19-Verstorbenen sicher durchführbar und für die medizinische Forschung hoch relevant.

\section{Schlüsselwörter}

Biomedizinische Forschung · Referenzstandards · Sicherheitsmanagement - SARS-CoV-2 . Ultrasonographie

\section{Practical aspects of COVID-19 autopsies}

\section{Abstract}

Background. The COVID-19 pandemic represents a so far unknown challenge for the medical community. Autopsies are important for studying this disease, but their safety was challenged at the beginning of the pandemic. Objectives. To determine whether COVID-19 autopsies can be performed under existing legal conditions and which safety standards are required.

Materials and methods. The autopsy procedure undertaken in five institutions in Germany, Austria, and Switzerland is detailed with respect to legal and safety standards. Results. In all institutions the autopsies were performed in technically feasible rooms. The personal equipment consisted of functional clothing including a disposable gown and apron, a surgical cap, eye protection, FFP-3 masks, and two pairs of gloves. In four institutions, complete autopsies were performed; in one institution the ultrasoundguided biopsy within the postmortal imaging and biopsy program. The latter does not allow the appreciation of gross organ pathology; however, it is able to retrieve standardized biopsies for diagnostic and research purposes. Several scientific articles in highly ranked journals resulted from these autopsies and allowed deep insights into organ damage and conclusions to better understand the pathomechanisms. Viral RNA was frequently detectable in the COVID-19 deceased, but the issue of infectivity remains unresolved and it is questionable if $\mathrm{Ct}$ values are greater than 30 . Conclusions. With appropriate safeguards, autopsies of people who have died from COVID-19 can be performed safely and are highly relevant to medical research.

\section{Keywords}

Biomedical research - Reference standards - Safety management - SARS-CoV-2 . Ultrasonography cherheit (nach gesetzlichen Vorgaben für biologische Sicherheit gemäß Biostoffverordnung und gemäß den geltenden Technischen Regeln für Biologische Arbeitsstoffe [TRBA 250 - Biologische Arbeitsstoffe im Gesundheitswesen und in der Wohlfahrtspflege]). Im Rahmen des DeRegCOVID hat die Registerzentrale in Aachen bislang gute Rückmeldungen zu dieser Empfehlung erhalten und konnte zusätzlich einige Zentren in Fragen der Arbeitssicherheit unterstützen (EMail: Covid.Pathologie@ukaachen.de).
Im Rahmen des Deutschen Forschungsnetzwerks für Autopsien bei Pandemien (DEFEAT PANDEMIcs) werden aktuell die Verfahrensempfehlungen weiter ausgearbeitet und durch das DeRegCOVID zur Verfügung gestellt. Aktuell bereits veröffentliche Empfehlungen sind in - Tab. 2 zusammengefasst.

\section{Gesetzliche Grundlagen der Obduktion und Corona- Regelungen}

\section{Deutschland}

In Deutschland ist die Regelung des Leichenschau- und Obduktionswesens Ländersache, sodass es keine bundesweite einheitliche Regelung gibt. Bundesrechtlich sind nur strafprozessuale, seuchengesetzliche und sozialversicherungsrechtliche Sektionen sowie in einem Teil 


\section{Schwerpunkt: COVID-19}

Tab. 2 Bisher veröffentlichte Empfehlungen für die Durchführung von COVID-19-Obduktionen

\begin{tabular}{l|l|l}
\hline Institution/Autor & Titel & Lan \\
\hline $\begin{array}{l}\text { Berufsverband der Deut- } \\
\text { schen Pathologen }\end{array}$ & $\begin{array}{l}\text { Vorsichtsmaßnahmen zur Durchführung von Autopsien in } \\
\text { SARS-, HIV-, Hepatitis-C- und Covid 19-Fällen }\end{array}$ & D \\
\hline
\end{tabular}

DE

schen Pathologen

SARS-, HIV-, Hepatitis-C- und Covid 19-Fällen

Boor et al., Aachen

Obduktion bei bestätigtem COVID-19-Todesfall

DE

Deutsche Gesellschaft
für Neuropathologie und
Neuroanatomie e. V.

Hinweise zur Prävention einer SARS-CoV-2-verursachten

Coronavirus-Krankheit 2019 (COVID-19) in medizinischen Untersuchungsstellen und Laboren

Deutsche Gesellschaft CNS-COVID19: Deutschlandweite Phänotypisierungsfür Neuropathologie und Plattform zur ZNS/PNS-Beteiligung von SARS-CoV-2 Neuroanatomie e. V.

German Biobanking

Node

Arbeiten mit SARS-CoV-2-Proben

DE

Österreichische Gesell- Obduktionen und COVID; Umgang mit an COVID-19 Verschaft für Pathologie/IAP storbenen

Austria

Schweizerische Gesell- Empfehlungen im Umgang mit SARS-CoV-2 für die Unterschaft für Rechtsmedizin suchung von Verstorbenen (Legalinspektion, postmortale Bildgebung und Autopsie) sowie für klinische Untersuchungen

Schweizerische UnfallVerhütung von Berufskrankheiten in pathologisch-anaversicherungsanstalt tomischen Instituten und histologischen Laboratorien

Royal College of Patholo- New briefing on COVID-19 - Autopsy practice relating to gists possible cases of COVID-19

WHO

WHO (2020) Transmission of SARS-CoV-2: implications for Interinfection prevention precautions
UK national

Link

https://www.pathologie.de/aktuelles/

corona/corona-detailansicht/?tx ttnews\%5Btt_news\%5D=1690\&

cHash=f7bb15ed3ab6d1ee006bcfbd87283316

Zusatzmaterial online, „Verfahrensanweisung COVID-19-Obduktionen der Uniklinik RWTH Aachen"

DE https://www.dgnn.de/images/documents/DGNNHinweise_zur_Praevention_COV19.pdf

DE https://jlubox.uni-giessen.de/dl/ fiAS1d59XB9qD1B8PvdFYwJj/CNS-COVID19Register_und_Asservierungsliste.pdf?inline https://www.bbmri.de/covid-19/arbeiten-mitsars-cov-2-proben/

https://oegpath.at/?s=COVID

https://www.sgrm.ch/inhalte/ForensischeMedizin/Empfehlungen_SGRM_SARS-CoV_ 25maerz2020.pdf

http://www.sohf.ch/Themes/Labo/2869_25_D.pdf

https://www.rcpath.org/uploads/assets/d5e28baf5789-4b0f-acecfe370eee6223/fe8fa85a-f0044a0c-81ee4b2b9cd12cbf/Briefing-on-COVID-19autopsy-Feb-2020.pdf

https://www.who.int/news-room/commentaries/ detail/transmission-of-sars-cov-2-implicationsfor-infection-prevention-precautions der Länder Sektionen bei Feuerbestattung geregelt. COVID-19-Obduktionen waren bislang überwiegend klinische Sektionen, für die ein Einverständnis des Verstorbenen zu Lebzeiten oder der Angehörigen erforderlich ist. An einigen Orten - allen voran in Hamburg wurden durch die Gesundheitsämter in Rahmen des Infektionsschutzgesetzes (IfSG) angeordnete Sektionen durchgeführt. Die gesetzlichen Grundlagen der Länder wurden und werden zumindest teilweise als unzureichend betrachtet [21, 22].

$\mathrm{Zu}$ Beginn der Pandemie in Deutschland erging vom Robert Koch-Institut die Empfehlung, Obduktionen von COVID19-Fällen zu vermeiden. Mittlerweile wurde diese Empfehlung, offensichtlich nicht zuletzt aufgrund einer Intervention des Bundesverbandes Deutscher Pathologen und der Deutschen Gesellschaft für Pathologie, wieder zurückgenommen. Bei der äußeren Leichenschau von an COVID-19 Verstorbenen sollten laut Robert Koch-Institut mindestens die Regelungen der Schutzstufe 3 nach der Biostoffverordnung eingehalten werden, insbesondere falls postmortale Maßnahmen, wie beispielsweise Leicheneröffnungen, durchgeführt werden. Für den Transport von Leichen werden keine besonderen Maßnahmen empfohlen. Tatsächlich geregelt wird dies allerdings wieder auf Länderebene, beispielsweise muss laut dem Landesamt für Gesundheit und Lebensmittelsicherheit in Bayern davon ausgegangen werden, dass jeder Verstorbene Träger von Krankheitserregern ist und damit potenziell infektiös sein kann. Aus diesem Grund ist beim Umgang mit einem Verstorbenen stets die nach $₫ 6$ Bestattungsverordnung (BestV) vorgegebene Schutzkleidung erforder- lich. Aus Vorsichtsgründen sollte in der Todesbescheinigung die Kennzeichnung als „infektiöse Leiche“ erfolgen. Dies zieht die nach $₫ 7$ BestV erforderlichen Maßnahmen nach sich. Letztere beinhalten den Transport der Leichen in sog. Bodybags. Der Leichentransport in einem von außen desinfizierten Bodybag, Tragen von Schutzkleidung und Angabe der möglichen, von dem oder der Verstorbenen ausgehenden Infektionsgefahr auf dem Totenschein sind in NordrheinWestfalen (NRW) gleichermaßen vorgeschrieben. Der Sarg ist mit der Angabe „infektiös“ oder „infektiöser Leichnam“ zu kennzeichnen. Das Aufbahren des Leichnams und Abschiednahme am offenen Sarg sind nicht gestattet (• Tab. 2).

Mittlerweile liegt auch eine Empfehlung zur Durchführung von Autopsien in SARS-CoV-2 verursachten Infektionsfällen vor [23]. 
Gemäß dem Zweiten Gesetz zum Schutz der Bevölkerung bei einer epidemischen Lage von nationaler Tragweite vom 19.05.2020 (Bundesgesetzblatt Jahrgang 2020 Teil I Nr. 23, ausgegeben zu Bonn am 22. Mai 2020) besteht für COVID-19 eine Arztmeldepflicht nach $\$ 6$ IfSG sowie für den direkten und indirekten Nachweis von SARS-CoV und SARS-CoV-2 eine Labormeldepflicht nach $₫ 7$ IfSG, soweit die Nachweise auf eine akute Infektion hinweisen.

\section{Österreich}

In Österreich sind Leichenschau und Obduktionen auf Ebene der Bundesländer gesetzlich geregelt (Leichenbestattungs- bzw. Bestattungsgesetze), auf Bundesebene die klinische Obduktion (\$ 25 Krankenanstalten- und Kuranstaltengesetz [KAKuG]: https://www.ris. bka.gv.at; Gesetzesnummer 10010285) sowie die Leichenbeschau und gerichtliche Obduktion im Rahmen der Strafprozessordnung. Im Gegensatz zu den anderen deutschsprachigen Ländern erfordert eine Obduktion in Österreich nur in Ausnahmefällen eine Zustimmung der Angehörigen. In öffentlichen Krankenhäusern besteht bei unklarer Todesursache zur Wahrung öffentlicher oder wissenschaftlicher Interessen, insbesondere wegen diagnostischer Unklarheit des Falles oder wegen eines vorgenommenen operativen Eingriffes, eine Obduktionspflicht. Außerdem müssen Obduktionen durchgeführt werden, wenn sie sanitätspolizeilich oder strafprozessual angeordnet wurden. Diese gesetzliche Bestimmung wird in die jeweilige Landesgesetzgebung übernommen (KAKuG der einzelnen Bundesländer), daher könnten grundsätzlich alle an COVID-19 Verstorbenen auf Basis der gesetzlichen Grundlagen ohne $\mathrm{Zu}$ stimmung von Angehörigen obduziert werden. Für die Durchführung der Obduktionen sind in den Krankenanstalten Institute für Pathologie zuständig, außerhalb der Krankenanstalten ebenfalls Pathologieinstitute (sanitätspolizeiliche Obduktionen) sowie Institute für $\mathrm{Ge}$ richtsmedizin (zum Teil sanitätspolizeiliche Obduktionen sowie gerichtliche Obduktionen). Obduktionen sind ferner in den jeweiligen Landesgesetzen über das Bestattungswesen (Leichenbestattungs- bzw. Bestattungsgesetze) geregelt, wobei dies detaillierter ausformuliert ist als in den KAKuG. Für die Durchführung von Obduktionen sind laut Ärztegesetz Fachärztinnen/Fachärzte für Pathologie oder Gerichtsmedizin zuständig.

$\mathrm{Zu}$ Beginn der Coronapandemie gab es von Seiten des Bundesministeriums für Soziales und Gesundheit weder eine Empfehlung für die Durchführung von Obduktionen an COVID-19-Verstorbenen noch eine Ablehnung. Die Österreichische Gesellschaft für Pathologie (ÖGPath/IAP Austria) hat als zuständige Fachgesellschaft auf ihrer Homepage (https://oegpath.at) auf die gesetzlichen Grundlagen und damit auf die Möglichkeit zur Obduktion hingewiesen. Die ersten Obduktionen fanden Mitte März in Wien (sanitätspolizeiliche Obduktion einer Verstorbenen in Heimquarantäne) und am LKH Graz II statt. Die Obduktionsfrequenz war regional sehr unterschiedlich und reichte von weniger als $1 \%$ bis zu $35 \%$ (Steiermark).

\section{Schweiz}

In der Schweiz obliegt das Obduktionswesen der kantonalen Gesundheitsgesetzgebung, stützt sich allerdings auf die Bundesgerichtsentscheide 97 I 221, 118 IV 319, 127 I 115, 129 I 173 (siehe https://www.bger.ch/ext/ eurospider/live/de/php/clir/http/index_ atf.php?lang=de) über die Rechte der Toten. In Basel-Stadt wird die klinische Obduktion durch das Gesundheitsgesetz (GesG, 300.100) IV.6.\$20 (siehe https://www.gesetzessammlung.bs.ch/ app/de/texts_of_law/300.100/versions/ 5117) geregelt und sieht die aktive Zustimmung (zu Lebzeiten) der verstorbenen Person bzw. der nächsten Angehörigen vor. Im Einklang mit dem Bundesgesetz über die Bekämpfung übertragbarer Krankheiten des Menschen, dem Epidemiengesetz (EpG) (siehe https://www.admin.ch/opc/de/ classified-compilation/20071012/index. html), Artikel 11 und Artikel 36 sieht das kantonale GesG $\$ 20$ im Punkt 3 vor, dass im Interesse der öffentlichen Gesundheit die Kantonsärztin oder der
Kantonsarzt auch gegen den Willen der entscheidungsbefugten Personen eine Obduktion anordnen kann. Ferner regelt das EpG über Artikel 25 die Sorgfaltspflicht beim Umgang mit Krankheitserregern alle erforderlichen Maßnahmen zu treffen, damit keine Menschen zu Schaden kommen können. Die Forschung an Verstorbenen wird schließlich über das Bundesgesetz über die Forschung am Menschen/ Humanforschungsgesetz (HFG) Kapitel 5 (siehe https://www.admin.ch/opc/ de/classified-compilation/20061313/ index.html\#id-5) geregelt und sieht eine aktive Zustimmung (zu Lebzeiten) der verstorbenen Person bzw. der nächsten Angehörigen vor und erlaubt, dass geringfügige Körpersubstanzmengen auch ohne Einwilligung zu Forschungszwecken anonymisiert asserviert werden, sofern keine dokumentierte Ablehnung der verstorbenen Person vorliegt.

Im Rahmen der COVID-19-Pandemie kam es in der Schweiz zu keiner Anwendung der in den kantonalen GesG vorgesehenen Passus, auch gegen den Willen der entscheidungsbefugten Personen Obduktionen anzuordnen. Autopsien wurden nach den üblichen gesetzlichen Rahmenbedingungen durchgeführt. Kantone bzw. Spitäler, welche aufgrund der Ausstattung ihrer Autopsieeinrichtungen den Anforderungen der EpG Artikel 25 nicht entsprechen konnten, sahen von Autopsien voll ab. So wurden z. B. im Kanton Tessin, welcher die höchste Mortalitätsrate im Rahmen der ersten Welle aufwies, keine Autopsien durchgeführt, während sie im Kanton Basel-Stadt bei $25 \%$ und im Kanton Basel-Landschaft bei $35 \%$ liegen.

\section{Obduktionstechnisches Vorgehen der verschiedenen Zentren}

\section{Augsburg}

Die technischen Voraussetzungen für einen sicheren Sektionsbetrieb waren in Augsburg gegeben. Der Luftwechsel im Sektionsraum betrug 13/h. Zusätzlich wurde ein Luftreinigungsgerät mit Virenfilter betrieben (s. - Tab. 1). Alle Obduktionen erfolgten durch ein Team 
von 4 Ärzten (2 Pathologen, 2 Internisten). Auf den Einsatz von Präparationsund Weiterbildungsassistenten wurde verzichtet, da es zunächst zwar theoretisch begründete Einschätzungen zur Infektionsgefahr im Sektionssaal gab, jedoch keine belastbaren Daten. Im Gegensatz zur allgemeinen Empfehlung, mit möglichst wenig Personal zu arbeiten, glauben wir hier einen Vorteil durch die Aufgabenteilung zu erreichen. Alle präparatorischen Aufgaben führten 2 Kollegen durch. Ein Kollege arbeitete als Dokumentar und Springer, der vierte kümmerte sich um die optimale Organ- und Gewebeasservierung. Die persönliche Schutzausstattung ist der - Tab. $1 \mathrm{zu}$ entnehmen. Die Sektionen erfolgten im Bodybag, in dem der Leichnam von der Station in die Prosektur verbracht wurde. Die Eröffnung des Thorax erfolgte mittels einer Knorpelschere, die des Schädels allerdings mit einer oszillierenden Säge. Der Einsatz einer oszillierenden Säge ohne Absaugeinrichtung blieb für die Obduzenten zwar folgenlos, wird jedoch nicht empfohlen. Ein besonderes Augenmerk richtete sich auf eine optimale Fixierung des entnommenen Gewebes mit ausreichendem Verhältnis von Gewebe zu Formalin (mind. 1:3). Die Fixierung des Lungengewebes wurde durch Injektion von ca. $300 \mathrm{ml}$ Formalin pro Lungenflügel mittels 50-ml-Perfusorspritzen und dicklumiger Injektionsnadel ins Parenchym unterstützt. Diese Technik hat sich in Augsburg auch bereits bei Lungenoperationspräparaten bewährt und führt hier zu besseren Ergebnissen als die bronchiale Instillation. Die minimale Fixationszeit betrug mindestens $96 \mathrm{~h}$. Die Gehirnobduktion erfolgte durch die neuropathologischen Kollegen der Technischen Universität München gemäß den Vorgaben der Deutschen Gesellschaft für Neuropathologie und Neuroanatomie (s. Tab. 2). Abstriche erfolgten aus dem Rachenraum, der Trachea und der Bronchien, in einzelnen Fällen auch der Bindehaut. Soweit möglich wurden auch Körperflüssigkeiten (Ergüsse, Liquor, Augenkammerwasser) asserviert. Im Verlauf wurden auch routinemäßig Gewebeproben kryoasserviert.
Nach Abschluss der Obduktion erfolgte ein Verschluss des Bodybags und eine Desinfektion der Hülle. Einmalschutzkleidung wurde in entsprechende Abwurfbehälter gegeben. Desinfektion und Abfallbeseitigung erfolgten durch geschultes Personal.

Alle 4 mit den Obduktionen betrauten Ärzte blieben gesund, was durch zweimalig durchgeführte Abstrichuntersuchungen und jeweils eine Antigenbestimmung bestätigt wurde.

\section{Erlangen}

Am Uniklinikum Erlangen wurde bislang (Stand 16.10.2020) bei 13 Verstorbenen eine COVID-19-Infektion nachgewiesen und in 4 Fällen eine Obduktion durchgeführt.

Alle Obduktionen wurden in einem separaten, für Risikosektionen vorgehaltenen Sektionsraum sowie mit adäquater persönlicher Schutzausrüstung der beteiligten Personen (s. ๑ Tab. 1) durchgeführt. Gemäß der zum Zeitpunkt der Obduktion geltenden Empfehlungen (die Obduktionen wurden zwischen April und Mai 2020 durchgeführt) wurde die Anzahl der beteiligten Personen so gering wie möglich gehalten. Die organpräparatorischen Arbeiten, die Asservierung und die initiale Dokumentation wurden durch einen erfahrenen Arzt in Weiterbildung, unterstützt durch einen medizinischen Präparator, durchgeführt. Die Dokumentation erfolgte dabei zunächst auf „kontaminierten“ Protokollbögen und wurde nach Abschluss der Obduktion auf neue, nicht kontaminierte Protokollbögen übertragen. Die Supervision oblag dem Prosektor (Facharzt). Die Obduktion erfolgte identisch wie in Augsburg.

Nach Abschluss der Obduktion erfolgte eine Desinfektion des Bodybags sowie anschließend die Verbringung in einen zweiten, frischen Bodybag, um einen maximalen Infektionsschutz zu erhalten. Die Einmalschutzkleidung wurde in entsprechende Abwurfbehälter gegeben. Reinigung und Desinfektion der wiederverwendbaren Schutzausrüstung, des Sektionssaales sowie Abfallentsorgung erfolgten durch geschultes Personal.
Die nativen Gewebeproben wurden kryoasserviert in, je nach Organgröße, großzügiger Menge in einem Kunststoffbehälter mit Schraubverschluss in einem gesonderten und gekennzeichneten Tiefkühlschrank $\left(-80^{\circ} \mathrm{C}\right)$. SARS-CoV-2 wurde durch den Ausschuss für Biologische Arbeitsstoffe (ABAS) in die Risikogruppe 3 und in die Schutzstufe 2 eingeordnet. Unter Einhaltung der entsprechenden Sicherheitsvorkehrungen sind ungerichtete Tätigkeiten (u. a. Probenlagerung) als unkritisch anzusehen [24].

Die histologischen Proben wurden bei Raumtemperatur für mindestens $48 \mathrm{~h}$ in $4 \%$ igem, gepuffertem Formalin fixiert.

\section{TU München}

Am Institut für Pathologie der Technischen Universität München (TUM) wurde zusammen mit der Ultraschallabteilung und Gewebebank (MTBIO) des Klinikums rechts der Isar ein durch postmortale Ultraschallbildgebung unterstütztes standardisiertes Verfahren für die Gewebegewinnung und -asservierung letaler COVID-19-Fälle etabliert, das „postmortal imaging and biopsy program“. Dieses berücksichtigt den Infektionsschutz für Mitarbeiter und die Qualitätssicherung postmortal entnommener Organproben und beantwortet diagnostische Fragen zur Pathobiologie und Gewebeschädigung von COVID-19. Das Verfahren und die Verwendung der Proben für die COVID-19-Forschung wurden von der Ethikkommission der TU München genehmigt.

Im Rahmen der Gelbfieberepidemie 2018 in Brasilien wurde ein minimalinvasives Verfahren, die sog. Minimalinvasive Autopsie (MIA) in einer etwas einfacheren Technik bereits erfolgreich angewendet [25], wobei Todesursachen mittels MIA mit vergleichbarer hoher Sicherheit wie mittels konventioneller Autopsie ermittelt werden konnten. Über erste positive Erfahrungen der minimal-invasiven Autopsie im Rahmen der COVID-19-Pandemie wurde bereits berichtet [26, 27]. Einer der Vorteile der minimal-invasiven Verfahren ist die fehlende Aerosolbildung, sodass unter Einhaltung der spezifischen Sicherheitsvorkehrungen (siehe - Tab. 1) 

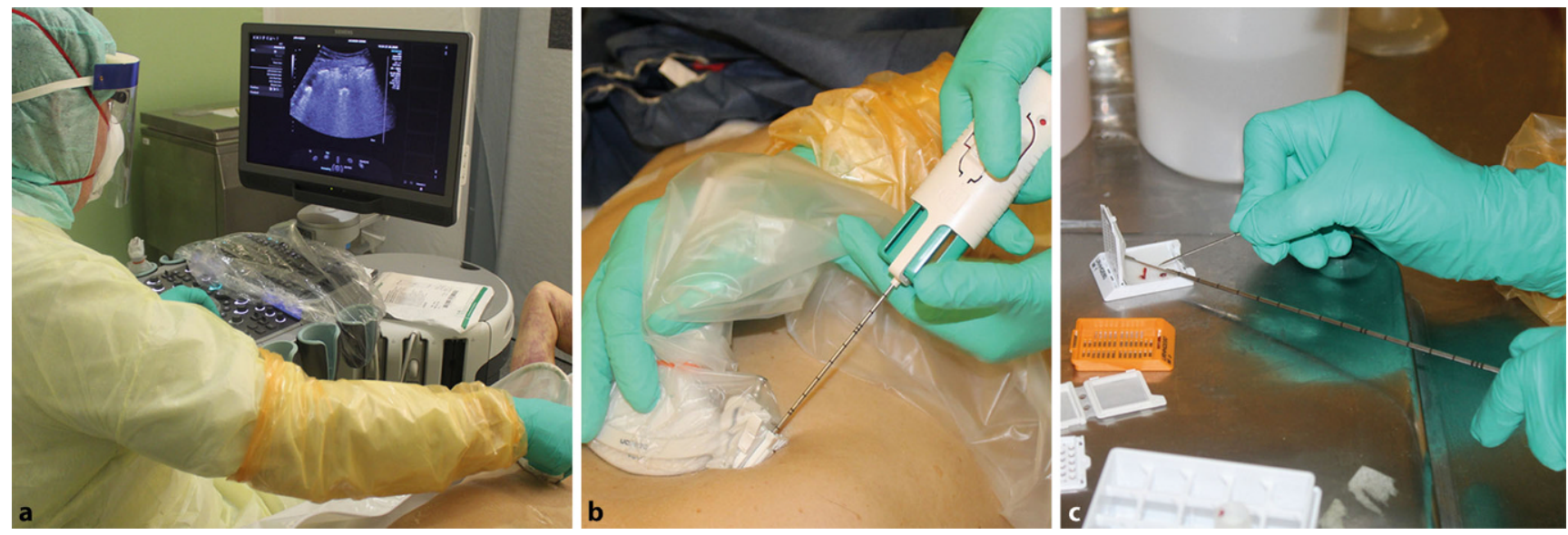

Abb. 1 A Minimal-invasive, ultraschallgesteuerte Autopsie (MIA-US). a Postmortale Sonografie. b Sonografisch gesteuerte Punktion unter Verwendung eines Schallkopfs mit Punktionsaufsatz. c Überführung der Stanzzylinder in Gewebekassetten zur weiteren Verarbeitung

bereits kurz nach Eintritt des Todes Gewebe für Untersuchungen auf zellulärer und subzellulärer Ebene sicher gewonnen werden kann. Um schnellstmöglich und treffsicher postmortales Gewebe zu asservieren, werden Organe bzw. Zielläsionen mit einem HighEnd-Ultraschallgerät identifiziert und mittels Punktionsaufsatz und Einmalbiopsiegerät unter Sichtkontrolle zielgenau punktiert (•Abb. 1). Für die Durchführung des Verfahrens wurde am Institut für Pathologie ein Punktionsraum im Sektionsbereich eingerichtet und mit ITAnbindung an das Klinikinformationssystem ausgerüstet. Das Team besteht aus einem fachärztlichen Internisten/einer fachärztlichen Internistin mit besonderer Ultraschallqualifikation (DEGUM III), einem Facharzt/einer Fachärztin für Pathologie und einem Mitarbeiter/einer Mitarbeiterin zur technischen Unterstützung des Probenmanagements. Bei Ableben eines COVID-19-Erkrankten am Klinikum rechts der Isar werden die Angehörigen des Verstorbenen informiert. Nach Einwilligung der Angehörigen wird unter Einhaltung der empfohlenen Infektionsschutzmaßnahmen (analog zur konventionellen Autopsie) das Verfahren im geöffneten Bodybag im Punktionsraum der Pathologie durchgeführt. Nach äußerer Leichenschau durch den Pathologen erfolgt nach standardisiertem Protokoll die Ultraschalluntersuchung der inneren Organe und die transkutane Punktion diver- ser Organe bzw. Zielläsionen (Trachea, Lunge, Herz, Leber, Pankreas, Nieren, Milz, Aorta, Lymphknoten u. a.). Mittels gezielter Schädeltrepanation wird weiterhin Hirngewebe und eine Knochenmarksprobe am vorderen Beckenkamm gewonnen. Die Ultraschalluntersuchung und die Punktionen werden in Rückenlage des Leichnams ohne Umlagerung durchgeführt, sodass eine mögliche umlagerungsbedingte Aerosolbildung verhindert wird. Je Organ bzw. Zielläsion werden mindestens 3 Proben gewonnen und in $4 \%$ gepuffertem Formalin (optimale Morphologie) bzw. dem formalinfreien PAXgene Tissue FIX (Qiagen N.V., Venlo, Niederlande; optimale Erhaltung von Morphologie und Molekülen) für $72 \mathrm{~h}$ fixiert (• Abb. 1). Zudem wird eine Probe je Organ/Zielläsion bei $-80^{\circ} \mathrm{C}$ eingefroren und in einer gesonderten und gekennzeichneten Tiefkühltruhe gelagert, sodass das gesamte Spektrum der Methoden der State-of-the-ArtPathologie für die postmortale Diagnostik COVID-19-Verstorbener sowie zur funktionellen Infektionsforschung zur Anwendung kommen kann.

Nach Abschluss der Obduktion erfolgt ein Verschluss und Desinfektion des Bodybags. Einmalschutzkleidung wird in entsprechende Abwurfbehälter entsorgt. Die Desinfektion und Abfallbeseitigung erfolgten durch geschultes Personal.

\section{Graz}

Zum Zeitpunkt der ersten Obduktionen Mitte März gab es kaum verfügbare Standards, außer jene der CDC (Centers for Disease Control and Prevention) in den USA und des Royal College of Pathologists des Vereinigten Königreichs $[28,29]$. Daher wurden rasch eigene Standards entwickelt und konsequent ausgeführt [30]. Bis zum 31.12.2020 wurden am Institut für Pathologie des LKH Graz II 104 COVID-19-Obduktionen nach identem Standard durchgeführt.

Im LKH Graz II besteht ein Obduktionsraum mit speziellem Belüftungssystem mit getrennter Abluft zur Verfügung (Zuluft von $2120 \mathrm{~m}^{3}$, Abluft von $2180 \mathrm{~m}^{3}$, Luftwechsel ca. 20fach/h), der als Airborne Infection Isolation Room (AIIR) klassifiziert ist. Alle Obduktionen wurden von einem 3-köpfigen Team (2 Pathologen und ein Obduktionsassistent) durchgeführt, die ersten 30 Obduktionen von demselben Ärztetandem. Die Sicherheitsmaßnahmen wurden vorab mit dem verantwortlichen Krankenhaushygieniker abgestimmt. Die Schutzmaßnahmen, insbesondere die Schutzkleidung, entsprach den Maßnahmen auf den COVID-19-Stationen, wobei das Pathologieteam an der hausinternen Schulung teilnahm (siehe • Tab. 1). Die Schutzkleidung bestand aus Funktionskleidung (Hose und Shirt), darüber ein OP-Mantel, eine Plastikschürze, 2 Paar Latexhandschuhe (bzw. ein schnittfester 


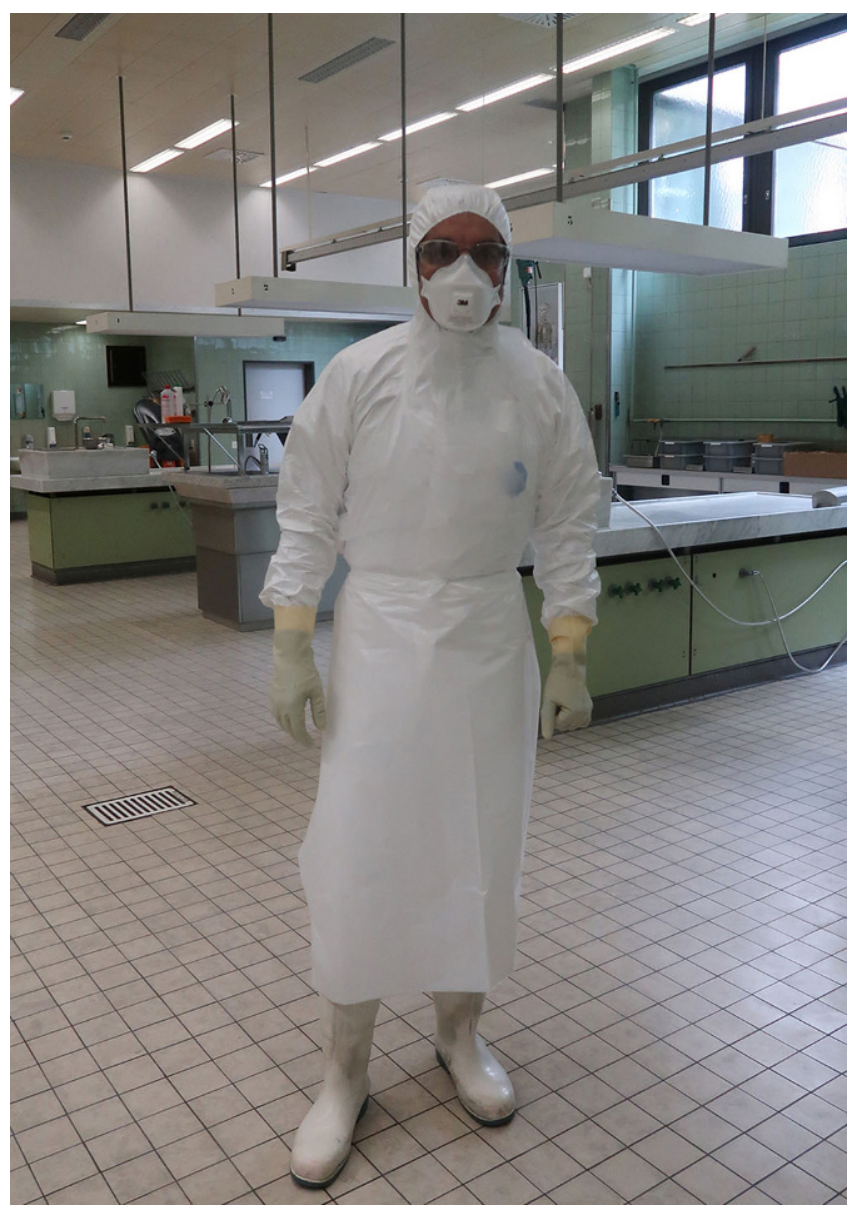

wurde in toto in Formalin fixiert und in fixiertem Zustand vom Apex zur Basis zur Entnahme von Proben für die histologische Untersuchung lamelliert. Die Abdominalorgane wurden in situ seziert und nach Präparation abgesetzt und abgewogen. Die tiefen Beinvenen wurden von dorsal nach Umdrehen der wieder verschlossenen Leiche präpariert. Aus allen Organen wurden Proben zur histologischen Untersuchung entnommen und außerdem Proben aus mehreren Organen eingefroren. Nach der Obduktion wurden die Verstorbenen in einem verschlossenen Bodybag untergebracht. Ausrüstung und Obduktionsraum wurden gemäß Hygieneplan mit Desinfektionslösungen gereinigt und das Werkzeug in einer speziellen Spülmaschine gewaschen. Alle an den Obduktionen beteiligten Personen (insgesamt 6 Ärzte und 2 Obduktionsassistenten) blieben gesund.

Abb. $2<$ Obduzent in Schutzausrüstung im Sektionssaal am Institut für Medizinische Genetik und Pathologie in Basel
Handschuh im Bereich der nichtmesserführenden Hand), Plastikgamaschen für die Arme, eine OP-Haube sowie eine FFP3-Maske. Für den Schutz der Augen wurde anfangs eine Schutzbrille verwendet, im Laufe der Zeit auf einen Schutzschild gewechselt, welches den gesamten Gesichtsbereich abdeckt und lokal produziert wird. Der OP-Mantel ist flüssigkeitsabweisend und durch die Reinigung in der Krankenhauswäscherei gut zu recyceln. Außerdem ist das Entkleiden sicherer als bei Plastikoveralls.

Einer der beiden Pathologen verblieb im sauberen Bereich und führte die Dokumentation durch, entnahm Abstriche und überwachte das hygienekonforme Verhalten des Obduzenten und des Obduktionsassistenten. Unter den Schutzmaßnahmen wurde speziell darauf geachtet, die Entstehung von Aerosolen zu vermeiden, insbesondere durch die Vermeidung von Wasserdampf. Daher wurde trocken obduziert, das Spülen mit Wasser vermieden und zur Reinigung ausschließlich eine Desinfektionslösung aus dem Desinfektionsspender verwendet. Vor der Obduktion wurde ein Abstrich aus dem Oropharynx entnommen, während der Obduktion getrennte Abstriche aus dem rechts- und linksseitigen Bronchialsystem sowie zum Teil aus anderen Organen. Die Obduktionstechnik wurde vor der ersten Obduktion festgelegt und bei allen Obduktionen angewandt. Für die Eröffnung des Gehirnschädels wurde eine elektrische Säge mit Absaugvorrichtung herangezogen. Das Gehirn wurde in toto entnommen und in Formalin fixiert, zuvor Hirnstamm und Kleinhirn abgetrennt und das Großhirn durch einen Frontalschnitt durch die Corpora mamillaria in 2 Teile getrennt. Bei 10 Obduktionen in der ersten Serie wurde aus Sicherheitsgründen auf die Gehirnentnahme verzichtet, in der Folge aber konsequent durchgeführt. Der Thorax wurde mit einer Knorpelzange eröffnet, Halsorgane und Thoraxsitus wurden en bloc entnommen. Die Lungen

\section{Basel}

In der Schweiz gibt es keine Empfehlungen für die Untersuchung von Verstorbenen mit SARS-CoV-2-Infektion bzw. COVID-19-Autopsien, weder von Seiten der Swiss National COVID-19 Science Task Force noch der Schweizerische Gesellschaft für Pathologie. Einzig die Schweizerische Gesellschaft für Rechtsmedizin empfiehlt eine FFP2-Maske für Autopsien, Schutzhandschuhe, ggf. schnittfeste Handschuhe, Schutzbrille oder Gesichtsschutz, wasserabweisende Überschürze (• Abb. 2; • Tab. 1), nach der Untersuchung gründliche Desinfektion aller Instrumente und Geräte sowie der Hände mit Standarddesinfektionsmittel und Entsorgung der Einwegmaterialien in einem separaten Sack [31].

Abgesteckt durch die o.g. Rahmenbedingungen und bei Verfügbarkeit eines Autopsiebereiches mit adäquater technischer Lüftung mit einem - wie von der Schweizerischen Unfallversicherungsanstalt empfohlen [32] - mindestens 6 fachem (im Fall Basel 9fachem) Luftwechsel pro Stunde und unter Einbezug der Erfahrung mit Autopsien bei HIV-, $\mathrm{HCV}$ - oder HBV-infizierten Individuen wurde im Basel am 16.03.2020 die erste Autopsie eines COVID-19-Verstorbenen 


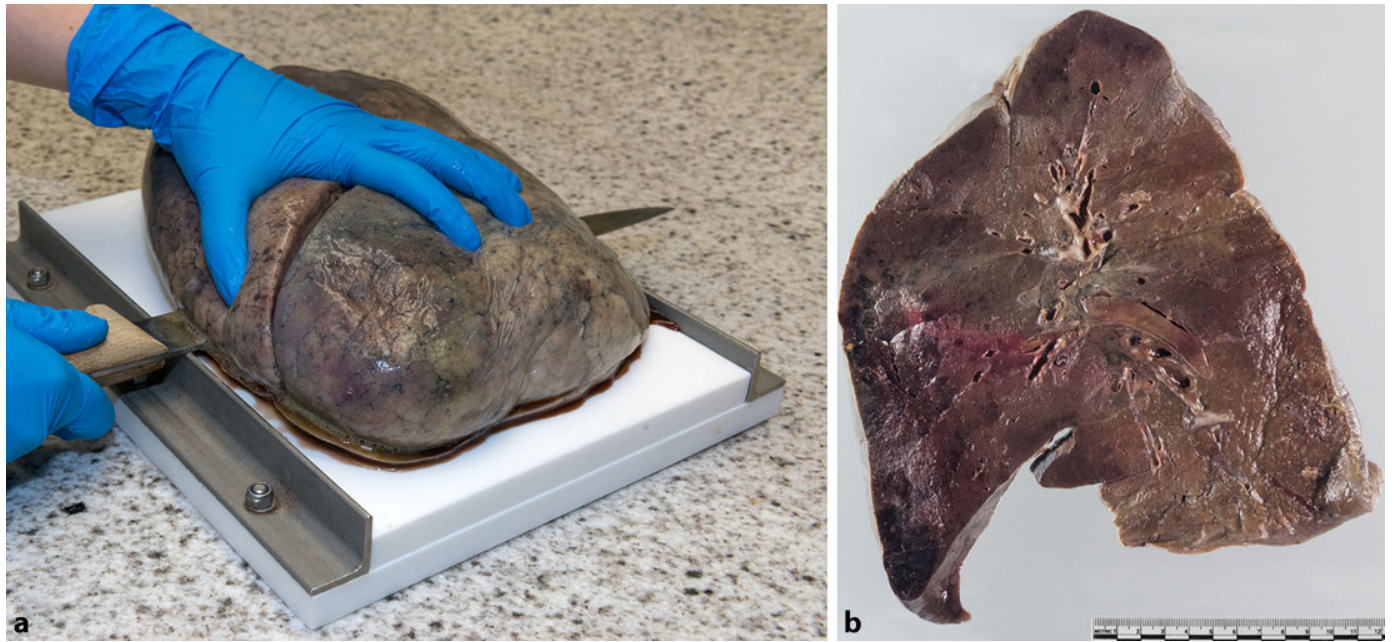

Abb. $3<$ Aufarbeitung eines fixierten Lungenflügels (Institut für Medizinische Genetik und Pathologie in Basel) mithilfe des sog. Lungenbretts (a) und parasagittalen, $10 \mathrm{~mm}$ dicken Scheiben (b) mit zum Zeitpunkt des Todes vorhandener (hoher) Viruslast durgeführt. Zu dieser Zeit waren die Richtlinien des Royal College of Pathologists [33] noch nicht publiziert (Erscheinungsdatum 20.03.3020), sondern nur auf der Internetseite der Gesellschaft zu finden [28]. Aus allen diesen Gründen war eigenverantwortliche Konzeptarbeit erforderlich. Unter der begründeten Annahme einer Tröpfchen- bzw. Schmierinfektion durch SARS-CoV-2 [34] und der potenziellen Infektiosität der nasalen und oralen Partie der Leichname, welche sich später als doch relevant erwiesen hat $[14,35,36]$, haben wir in Basel beschlossen 1) ca. $2 \mathrm{~h}$ vor der Autopsie $4 \%$ iges gepuffertes Formalin (100-200 ml) mittels Pipetten in die Nase und in dem Mund der Leichname zu instillieren, ferner - zur weiteren Reduktion der Infektiosität - 2) das Hantieren an der Leiche bzw. mit Organen in die Hände von 2 Fachärzten zu geben (MT und AT; beide während der ganzen Zeit der Pandemie bis zum heutigen Tag gesund), 3) bei Ausfall der Präparatoren (was auch eingetroffen ist) eine In-corpore-Autopsietechnik, analog derer, die in einigen Instituten für Rechtsmedizin benutzt wird, anzuwenden [2], 4) Hirnautopsien nur bei Patienten jünger als 75 oder mit neurologischer Symptomatik durchzuführen, 5) das Neurokranium ausschließlich mit der Handsäge zu eröffnen und 6) das Trachea-LungenOrganpaket mit kaltem (bessere Penetration) $4 \%$ igem gepuffertem Formalin (2-4l) zu infundieren, anschließend mit der Kocherklemme zu blockieren und eingetaucht in Formalin für $72 \mathrm{~h}$ fixieren zu lassen. Die Trachea wurde anschließend gewöhnlich präpariert und untersucht, während die Lungen mithilfe des sog. Lungenbretts (• Abb. 3a) in $10 \mathrm{~mm}$ dicken parasagittalen Schnitten (beste Ebene zur Erhaltung des Zusammenhangs der Lappen) aufgearbeitet wurden (- Abb. 3b). Es wurde systematisch und reichlich (62 Standardkassetten) Gewebe zur Klärung der Todesursache und Virusausbreitung asserviert. Die Qualität der Asservate ermöglichte es uns und auch in Zusammenarbeit mit internationalen Partnern, wichtige und später unabhängig breit bestätigte Erkenntnisse über die Pathogenese von COVID19 als generalisiertes, dennoch pulmozentrisches mikroangiopathisches/ endotheliales Dysfunktionssyndrom mit schwerer thrombotischer Diathese $\mathrm{zu}$ gewinnen $[2,7,18,37,38]$.

\section{Zusammenfassung und Diskussion}

Bereits anhand einer geringen Fallzahl konnten von unseren Teams hochkarätige wissenschaftliche Arbeiten veröffentlicht und somit wesentliche Beiträge zu einem besseren Verständnis der Erkrankung geliefert werden. Dies unterstreicht die Notwendigkeit von Obduktionen in der Situation einer neu aufgetretenen Krankheit, im speziellen Fall einer Infektionserkrankung. Anzahl und Umfang der Obduktionen sowie auch die Schutzmaßnahmen unterscheiden sich zwischen den Zentren, außerdem sind die gesetzlichen Grundlagen in den 3 Ländern unterschiedlich. Basel kann ein Modell der minimalen Personalexposition darstellen, während die TU München das Spektrum technischer Möglichkeiten autoptischer Verfahren erweitert, um mittels postmortaler Ultraschallbildgebung und Punktion sicher und standardisiert Gewebeproben für postmortale Diagnostik und Forschung zu gewinnen.

Von verschiedenen wissenschaftlichen Organisationen und Berufsverbänden liegen Empfehlungen für die Obduktionen bei COVID-19 vor, die maßgeblich die Handlungsfähigkeit der mit Autopsien betrauten Fächer unterstützten. Eine einheitliche, detaillierte und verbindliche Empfehlung fehlt allerdings bislang. Für Deutschland wird eine solche derzeit im Rahmen des vom Bundesministerium für Forschung und Bildung geförderten Deutschen Forschungsnetzwerks für Autopsien in Pandemien (DEFEAT PANDEMIcs; siehe Artikel in dieser Sonderausgabe) erarbeitet.

Die Frage nach der Infektionsgefahr im Rahmen der COVID-19-Obduktionen konnte bisher nicht umfassend beantwortet werden. Einzelne Untersuchungen haben gezeigt, dass Virus-RNA bei Verstorbenen zwar nachweisbar ist [11], die meist hohen Ct-Werte (über 30) eine Infektiosität jedoch infrage stellen. Am Institut in Augsburg wurde in 50 konstitutiven postmortalen Analysen aus Rachen und Atmungswegen im Mittel ein Ct-Wert von $23 \pm 6$ ermittelt (unpubl. 
Daten). Eine Anzüchtung von Viren aus Abstrichen von Verstorbenen ist in einzelnen Fällen gut dokumentiert [35, 39]. Es liegen hier allerdings keine gleichzeitigen quantitativen Informationen über die Virus-RNA in Form von Ct-Werten vor [36]. Ganz wesentlich ist die Vermeidung der Aerosolbildung durch eine möglichste trockene Obduktionstechnik sowie der Schutz durch adäquate Atemschutzmasken (idealerweise FFP3). In diesem Rahmen ist wichtig festzuhalten, dass es bisher keine Berichte von sicher im Obduktionssaal erworbenen Infektionen an SARS-CoV-2 gibt. Unsere Mitarbeiter und Mitarbeiterinnen blieben gesund und frei von einer Infektion. Eine Zurückhaltung gegenüber Obduktionen basiert damit auf keiner rationalen Grundlage.

\section{Fazit für die Praxis}

- Obduktionen stellen einen unverzichtbaren Teil der Erforschung von neuen Erkrankungen, wie z. B. COVID19, dar.

- Standards einschließlich SOPs wurden von mehreren Instituten unabhängig voneinander entwickelt, vielfach reproduziert und zum Teil publiziert. Darüber hinaus sollten Standards offiziell anerkannt werden. Die minimal-invasive, ultraschallgesteuerte Autopsie (MIA-US) stellt eine innovative Ergänzung zur kompletten Obduktion dar, mit dem Nachteil, makroskopische Veränderungen nur eingeschränkt erfassen zu können.

- Dem Schutz des mit COVID-19Obduktionen betrauten Personals durch technische Voraussetzungen (ausreichender Luftwechsel) und geeignete Schutzausrüstung gilt besonderes Augenmerk.

- Unter Beachtung der erforderlichen Schutzmaßnahmen sind COVID-19Obduktionen sicher durchführbar.

\section{Korrespondenzadresse}

\section{Prof. Dr. med. Bruno MärkI}

Institut für Pathologie und Molekulare Diagnostik, Universitätsklinikum Augsburg Stenglinstr. 2, 86156 Augsburg, Deutschland bruno.maerkl@uka-science.de
Danksagung. Diese Arbeit wurde unterstützt durch das Deutsche Register für COVID-19 Autopsien (DeRegCOVID, www.DeRegCOVID.ukaachen.de), finanziert durch das Bundesministerium für Gesundheit (ZMVI1-2520COR201) und das Bundesministerium für Bildung und Forschung im Rahmen des Netzwerkes Universitätsmedizin (DEFEAT PANDEMIcs, 01KX2021). Unser Dank gilt den Kollegen und Kolleginnen der einzelnen Pathologien und Neuropathologien sowie denen der klinischen Fächer, die uns in mannigfaltiger Form unterstützt haben. Aus München sei besonders PD Dr. med. vet. Katja Steiger und PD. Dr. med. Konrad Stock gedankt. Frau Dr. Jasmin Haslbauer, Herrn Ralph Schoch und Herrn Thomas Rost aus Basel sei herzlich für die unschätzbare und tatkräftige Unterstützung hinsichtlich COVID-19 Autopsien gedankt: amici certi in re incerta cernitur. Diese Arbeit ist den Teams aus Ärztinnen und Ärzten und Pflegekräften gewidmet, die täglich um das Leben der COVID-Patienten kämpfen. Daneben gilt unser besonderer Dank den Angehörigen der Verstorbenen, die in der Situation des Verlustes und der Trauer durch Ihre Einverständnisse einen unschätzbaren Beitrag zur Erforschung dieser in vieler Hinsicht bedrohlichen Erkrankung geleistet haben.

\section{Einhaltung ethischer Richtlinien}

Interessenkonflikt. P. Boor, P. Eichhorn, A. Hartmann, S. F. Lax, B. Märkl, T. Menter, K.Skok, J. Slotta-Huspenina, S. von Stillfried, A. Tzankov und G. Weirich geben an, dass kein Interessenkonflikt besteht.

Für diesen Beitrag wurden von den Autoren keine Studien an Menschen oder Tieren durchgeführt. Für die aufgeführten Studien gelten die jeweils dort angegebenen ethischen Richtlinien.

\section{Literatur}

1. Lax SF, Skok K, Zechner P, Kessler HH, Kaufmann N, Koelblinger C, Vander K, Bargfrieder U, Trauner M (2020) Pulmonary Arterial Thrombosis in COVID19 With Fatal Outcome: Results From a Prospective, Single-Center, Clinicopathologic Case Series. Ann Intern Med 173(5):350-361. https://doi.org/10. 7326/M20-2566

2. Menter T, Haslbauer JD, Nienhold R, Savic $S$, Hopfer H, Deigendesch N, Frank S, Turek D, Willi N, Pargger H, Bassetti S, Leuppi JD, Cathomas G, Tolnay M, Mertz KD, Tzankov A (2020) Postmortem examination of COVID-19 patients reveals diffuse alveolar damage with severe capillary congestion and variegated findings in lungs and other organs suggesting vascular dysfunction. Histopathology. https://doi.org/10.1111/his.14134

3. Wichmann $D$, Sperhake JP, Lutgehetmann $M$ Steurer $S$, Edler $C$, Heinemann A, Heinrich $F_{\text {, }}$ Mushumba H, Kniep I, Schroder AS, Burdelski C, de Heer G, Nierhaus A, Frings D, Pfefferle S, Becker H, Bredereke-Wiedling $\mathrm{H}$, de Weerth $\mathrm{A}$, Paschen $\mathrm{HR}$, Sheikhzadeh-Eggers S, Stang A, Schmiedel S, Bokemeyer C, Addo MM, Aepfelbacher M, Puschel K, Kluge S (2020) Autopsy Findings and Venous Thromboembolism in Patients With COVID-19: A Prospective Cohort Study. Ann Intern Med 173 (4):268-277. https://doi.org/10.7326/ M20-2003

4. Barton LM, Duval EJ, Stroberg E, Ghosh S, Mukhopadhyay S (2020) COVID. USA. Am J Clin
Pathol, Oklahoma https://doi.org/10.1093/ajcp/ aqaa062 (19 Autopsies)

5. Tian $\mathrm{S}$, Hu W, Niu L, Liu $\mathrm{H}, \mathrm{Xu} \mathrm{H}$, Xiao SY (2020) Pulmonary Pathology of Early-Phase 2019 Novel Coronavirus (COVID-19) Pneumonia in Two Patients With Lung Cancer. J Thorac Oncol 15(5):700-704. https://doi.org/10.1016/j.jtho. 2020.02.010

6. Tian S, Xiong Y, Liu H, Niu L, Guo J, Liao M, XiaoSY (2020) Pathological study of the 2019 nove coronavirus disease (COVID-19) through postmortem core biopsies. Mod Pathol 33(6):1007-1014. https://doi.org/10.1038/s41379-020-0536-x

7. Ackermann M, Verleden SE, Kuehnel M, Haverich A, Welte T, Laenger F, Vanstapel A, Werlein C, Stark H, Tzankov A, Li WW, Li VW, Mentzer SJ, Jonigk D (2020) Pulmonary Vascular Endothelialitis, Thrombosis, and Angiogenesis in Covid-19. N Engl J Med 383(2):120-128. https://doi.org/10.1056/ NEJMoa2015432

8. Varga Z, Flammer AJ, Steiger $P$, Haberecker $M$, Andermatt R, Zinkernagel AS, Mehra MR, Schuepbach RA, Ruschitzka F, Moch H (2020) Endothelial cell infection and endotheliitis in COVID-19. Lancet 395(10234):1417-1418. https://doi.org/10.1016/ S0140-6736(20)30937-5

9. Haslbauer JD, Perrina V, Matter M, Dellas A, Mihatsch MJ, Tzankov A (2020) Retrospective Postmortem SARS-CoV-2 RT-PCR of Autopsies with COVID-19-Suggestive Pathology Supports the Absence of Lethal Community Spread in Basel, Switzerland, before February 2020. Pathobiology. https://doi.org/10.1159/000512563

10. Lax SF, SkokK, Trauner M (2020) Pulmonary arterial thrombosis as an important complication of COVID-19 pulmonary disease: letter to the editor. Virchows Arch 477(3):467-468. https://doi.org/10 1007/s00428-020-02896-4

11. Su H, Yang M, Wan C, Yi LX, Tang F, Zhu HY, Yi F, Yang HC, Fogo AB, Nie X, Zhang C (2020) Renal histopathological analysis of 26 postmortem findings of patients with COVID-19 in China. Kidney Int 98(1):219-227. https://doi.org/10.1016/j.kint. 2020.04.003

12. Puelles VG, Lutgehetmann M, Lindenmeyer MT, Sperhake JP, Wong MN, Allweiss L, Chilla $S$, Heinemann A, Wanner N, Liu S, Braun F, Lu S, Pfefferle S, Schroder AS, Edler C, Gross O, Glatzel M, Wichmann D, Wiech T, Kluge S, Pueschel K, Aepfelbacher M, Huber TB (2020) Multiorgan and Renal Tropism of SARS-CoV-2. N Engl J Med 383(6):590-592. https://doi.org/10.1056/ NEJMc2011400

13. Amann K, Boor P, Wiech T et al. (2021) COVID-19Auswirkungen auf die Niere. Pathologe. https:// doi.org/10.1007/s00292-020-00899-1

14. SkokK, StelzIE, TraunerM, Kessler HH, Lax SF (2020) Post-mortem viral dynamics and tropism in COVID19 patients in correlation with organ damage. Virchows Arch. https://doi.org/10.1007/s00428 020-02903-8

15. Meinhardt J, Radke J, Dittmayer C, Mothes R, Franz J, Laue $M$, Schneider J, Brünink S, Hassan O, Stenzel W, Windgassen M, Rößler L, Goebel H-H, Martin $\mathrm{H}$, Nitsche A, Schulz-Schaeffer WJ, Hakroush S, Winkler MS, Tampe B, Elezkurtaj $S$, Horst D, Oesterhelweg L, Tsokos M, Heppner BI, Stadelmann C, Drosten C, Corman VM, Radbruch H, Heppner FL (2020) Olfactory transmucosal SARSCoV-2 invasion as port of Central Nervous System entry in COVID-19. Patients Prepr. https://doi.org/ 10.1101/2020.06.04.135012

16. Matschke J, Lutgehetmann M, HagelC, SperhakeJP, Schroder AS, Edler C, Mushumba H, Fitzek A, All- 
weiss L, Dandri M, Dottermusch M, Heinemann A, Pfefferle S, Schwabenland M, Sumner Magruder $D_{,}$ Bonn S, Prinz M, GerloffC, Puschel K, Krasemann S, Aepfelbacher M, Glatzel M (2020) Neuropathology of patients with COVID-19 in Germany: a post-mortem case series. Lancet Neurol 19(11):919-929. https://doi.org/10.1016/S1474-4422(20)30308-2

17. Pezzini A, Padovani A (2020) Lifting the mask on neurological manifestations of COVID-19. Nat Rev Neurol. https://doi.org/10.1038/s41582-0200398-3

18. Deigendesch N, Sironi L, Kutza M, Wischnewski S, Fuchs V, Hench J, Frank A, Nienhold R, Mertz KD, Cathomas G, Matter MS, Siegemund M, Tolnay M, Schirmer L, Probstel AK, Tzankov A, Frank S (2020) Correlates of critical illness-related encephalopathy predominate postmortem COVID-19 neuropathology. Acta Neuropathol. https://doi. org/10.1007/s00401-020-02213-y

19. Acker T (2021) Covid-19-Auswirkungen auf das ZNS. Pathologe. https://doi.org/10.1007/s00292021-00924-x

20. von Stillfried $S$, Bulow RD, Rohrig R, KnuchelClarke R, Boor P, DeRegCovid (2020) Autopsy registry can facilitate COVID-19 research. EMBO Mol Med 12(8):e12885. https://doi.org/10.15252/ emmm.202012885

21. Dettmeyer R, Madea B (2002) Obduktionen: Unsichere und uneinheitliche. Rechtslage Dtsch Arztebl Intern 99(36):2311-2314

22. Edler C, Schröder AS, Aepfelbacher M, Fitzek A, Heinemann A, Heinrich F, Klein A, Langenwalder $F$, Lutgehetmann M, Meissner K, Püschel K, Schadler J, Steurer S, Mushumba H, Sperhake JP (2020) Dying with SARS-CoV-2 infection-an autopsy study of the first consecutive 80 cases in Hamburg, Germany. Int Jegal Med 134(4):1275-1284. https://doi.org/ 10.1007/s00414-020-02317-w

23. Bundesverband-deutscher-Pathologen (2020) Vorsichtsmaßnahmen zur Durchführung von Autopsien in SARS-, HIV-, Hepatitis-C- und Covid 19-Fällen. https://www.pathologie.de/aktuelles/ corona/corona-detailansicht/?tx ttnews\%5Btt news $\% 5 \mathrm{D}=1690 \& \mathrm{cHash}=$ f7bb15ed3ab6d1ee006 bcfbd87283316.Zugegriffen: 1.Nov. 2020

24. German-Biobank-Node (2020) Arbeiten mit SARSCoV-2-Proben. https://www.bbmri.de/covid-19/ arbeiten-mit-sars-cov-2-proben/. Zugegriffen: 28. Nov. 2020

25. Duarte-Neto AN, Monteiro RAA, Johnsson J, Cunha MDP, Pour SZ, Saraiva AC, Ho YL, da Silva LFF, MauadT,Zanotto PMA, Saldiva PHN, de Oliveira IRS, Dolhnikoff M (2019) Ultrasound-guided minimally invasive autopsy as a tool for rapid post-mortem diagnosis in the 2018 Sao Paulo yellow fever epidemic: Correlation with conventional autopsy. PLoSNegl Trop Dis 13(7):e7625. https://doi.org/10. 1371/journal.pntd.0007625

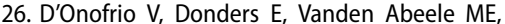
Dubois J, Cartuyvels R, Achten R, Lammens M, Dendooven A, Driessen A, Augsburg L, Vanrusselt J, Cox J (2020) The clinical value of minimal invasive autopsy in COVID-19 patients. PLoS ONE 15(11):e242300. https://doi.org/10.1371/journal. pone. 0242300

27. Duarte-Neto AN, Monteiro RAA, da Silva LFF, Malheiros $D$, de Oliveira EP, Theodoro-Filho J, Pinho JRR, Gomes-Gouvêa MS, Salles APM, de Oliveira IRS, Mauad T, Saldiva PHN, Dolhnikoff M (2020) Pulmonary and systemic involvement in COVID-19 patients assessed with ultrasound-guided minimally invasive autopsy. Histopathology 77(2):186-197.https://doi.org/10.1111/his.14160
28. Osborn M, Lucas S, Stewart R, Swift B, Youd E (2020) Briefing on COVID-19: Autopsy practice relating to possible cases of COVID-19 (2019-nCov novel coronavirus from China 2019/2020). https:// www.rcpath.org/uploads/assets/d5e28baf-57894b0f-acecfe370eee6223/fe8fa85a-f004-4a0c81ee4b2b9cd12cbf/Briefing-on-COVID-19autopsy-Feb-2020.pdf. Zugegriffen: 1.Nov. 2020

29. Centers_for_Disease_Control_and_Prevention (2021) Collection and Submission of Postmortem Specimens from Deceased Persons with Confirmed or Suspected COVID-19. https://www. cdc.gov/coronavirus/2019-ncov/hcp/guidancepostmortem-specimens.html. Zugegriffen: 10. Jan. 2021

30. Skok K, Vander K, Setaffy L, Kessler HH, Aberle S, Bargfrieder U, Trauner M, Lax SF (2020) COVID-19 autopsies: Procedure, technical aspects and cause of fatal course. Experiences from a. Single-center Pathol Pract. https://doi.org/10.1016/j.prp.2020. 153305

31. Medizin SGfR-F (2020) Empfehlungen im Umgang mit SARS-CoV-2 für die Untersuchung von Verstorbenen (Legalinspektion, postmortale Bildgebung und Autopsie) sowie für klinische Untersuchungen. https://www.sgrm.ch/ inhalte/Forensische-Medizin/Empfehlungen_ SGRM_SARS-CoV_25maerz2020.pdf. Accessed 1. Nov. 20202020

32. Jost $M$, Rüegger $M$, Gutzwiller $A$, Liechti $B$, Wolf R (2003) Verhütung von Berufskrankheiten in pathologisch-anatomischen Instituten und histologischen Laboratorien. www.sohf.ch/Themes/ Labo/2869_25_D.pdf.Zugegriffen:1.Nov. 2020

33. Hanley B, Lucas SB, Youd E, Swift B, Osborn M (2020) Autopsy in suspected COVID-19 cases. J Clin Pathol 73(5):239-242. https://doi.org/10.1136/jclinpath2020-206522

34. WHO (2020) Transmission of SARS-CoV-2: implications for infection prevention precautions. https:// www.who.int/news-room/commentaries/detail/ transmission-of-sars-cov-2-implications-forinfection-prevention-precautions. Accessed 1. Nov. 20202020

35. Plenzig S, Held $H$, Gradhand $E$, Kettner $M$, Verhoff MA, Berger A (2020) Infektiosität von COVID-19-Leichen. Rechtsmedizin. Berl 5:365

36. Basso C, Calabrese F, Sbaraglia M, Del Vecchio C, Carretta G, Saieva A, Donato D, Flor L, Crisanti A, Dei Tos AP (2020) Feasibility of postmortem examination in the era of COVID-19 pandemic: the experience of a Northeast Italy University Hospital. Virchows Arch 477(3):341-347. https://doi.org/10. 1007/s00428-020-02861-1

37. Löffler KU, Reinhold A, Herwig-Carl MC, Tzankov A Holz FG, Scholl HPN, Meyer P (2020) Ocular post-mortem findings in patients having died from COVID-19. Ophthalmologe 117(7):648-651. https://doi.org/10.1007/s00347-020-01149-8

38. Eckermann M, Frohn J, Reichardt M, Osterhoff M, Sprung M, Westermeier F, Tzankov A, Werlein C, Kühnel M, Jonigk D, Salditt T (2020) 3D virtual pathohistology of lung tissue from Covid19 patients based on phase contrast X-ray tomography. Elife. https://doi.org/10.7554/eLife. 60408

39. Borczuk AC, Salvatore SP, Seshan SV, Patel SS, Bussel JB, Mostyka M, Elsoukkary S, He B, Del Vecchio C, Fortarezza F, Pezzuto F, Navalesi P, Crisanti A, Fowkes ME, Bryce $\mathrm{CH}$, Calabrese $\mathrm{F}$, Beasley MB (2020) COVID-19 pulmonary pathology: a multiinstitutional autopsy cohort from Italy and New York City. Mod Pathol. https://doi.org/10.1038/ s41379-020-00661-1
Der besondere Fall - Gestalten Sie Der Pathologe mit

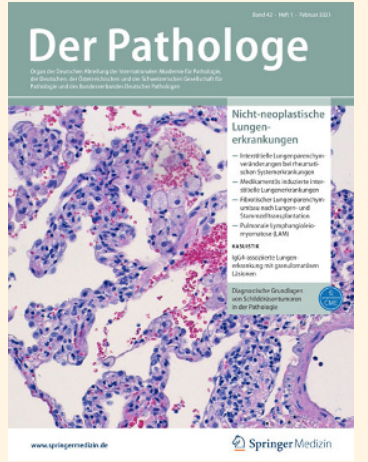

Sehr geehrte Autor:innen,

wir freuen uns, wenn Sie die Zeitschrift Der Pathologe mitgestalten. Zeigen Sie anhand von kurzen Fallbeispielen Besonderheiten der ärztlichen Praxis, Fallstricke in der Diagnostik und ungewöhnliche Krankheitsverläufe auf. Vermitteln Sie wertvolle Hinweise zu Diagnostik und Therapie. Im Mittelpunkt steht Ihr Bildmaterial, anhand dessen die Leser:innen eine Diagnose stellen und prüfen können, ob sie richtig lagen. Der Herausgeber der Rubrik Der besondere Fall, Herr Prof. W. Saeger (Hamburg), begutachtet die eingereichten Arbeiten und garantiert so die erforderliche Qualität.

\section{Checkliste zur Manuskripterstellung:}

- Text bitte als Datei schicken (.doc)

- Anschrift der Korrespondenzadresse mit E-Mail sowie Portraitfoto

- Gesamtumfang: max. 10.000 Zeichen inkl. Leerzeichen

- Kurzer, prägnanter Beitragstitel (dt. und engl., ggf. erläuternder Untertitel)

- Gliederung: Anamnese, Befunde, Diagnose, Verlauf und Therapie, Diskussion

- Kurze Zwischenüberschriften

- Fazit für die Praxis (max. 500 Zeichen)

- 3-4 Abbildungen in Erstpublikation

Bitte reichen Sie Ihren Beitrag im Editorial Manager ein:

www.editorialmanager.com/depa 